www revistadeempresafamiliar.uma.es

\title{
El consejero en la empresa familiar: teoría y praxis
}

\section{Family business advisor: theory and praxis}

\author{
Maribel Rodríguez Zapatero ${ }^{\mathrm{a}, *} \cdot$ Magdalena Rodriguez Jiménez ${ }^{\mathrm{b}} \cdot$ José Javier Rodriguez Alcaide $^{\mathrm{c}}$ \\ ${ }^{a}$ Departamento de Estadística, Econometría, Investigación Operativa y Organización de Empresas. \\ Universidad de Córdoba,14005 (Spain) \\ ${ }^{b, c}$ Cátedra Prasa de Empresa Familiar. Universidad de Córdoba,14005 (Spain)
}

D A T OS A R TÍC UL O

Historial:

Recibido 23-02-2012

Aceptado 23-05-2012

Palabras clave:

Empresa Familiar

Consejero

Códigos JEL:

L10, M20

\section{R E S U M E N}

El presente trabajo de investigación, basado en más de diez años de experiencia en asesoramiento a la empresa familiar, tiene por objeto clarificar el papel que debe jugar el asesor de una empresa familiar, dotar a éste de herramientas eficaces y eficientes para el desarrollo de su trabajo con el fin último de ayudar a la familia empresaria en su traspaso generacional. Se realiza un recorrido sobre toda la etapa metodológica común a todas las familias empresarias que por diferentes y muy variados motivos deciden comenzar el camino del protocolo o pacto familiar. Ponemos de manifiesto aquellas herramientas que han resultado de mayor utilidad al asesor para descubrir el complicado mundo de las relaciones interpersonales que tienen lugar en el seno de la familia empresaria y que sin lugar a dudas condicionan su supervivencia.

\section{A B S T R A C T}

This research paper aims to provide insights about the role the family business adviser must developed to obtain positive results helping the family business agreements process (protocol). The experiences shown in this paper are based in more than ten years of research in family business field. All the methodological phases applied to these family businesses, that decided signing a protocol are shown. We expose some of the experiences lived within these family business. We point out those tools that were most valuable for family business adviser to achieve a better comprehension of the complicated relationship that takes place within the family business members.

\footnotetext{
Autor de contacto.

Correoselectrónicos:es3rozai@uco.es,pu2rojim@uco.es,palroalj@uco.es
} 


\section{Introducción}

En este trabajo ponemos a la luz el modelo metodológico de asesoramiento a la empresa familiar que hemos empleado en la Cátedra de Empresa Familiar de la Universidad de Córdoba. Este modelo es fruto de la experiencia en investigación y asesoramiento a las familias empresarias durante más de once años. Escribimos sobre el mismo con el objetivo de ayudar a otros consejeros de empresa familiar en la complicada tarea de asesoramiento a la familia empresaria.

Entenderemos por consejero aquella persona que trabaja conjuntamente con los miembros de la familia empresaria-organización para encarar situaciones, es la persona que ayuda a la familia a entender el sistema de modo holístico (familia-propiedad-empresa), su dinámica y el modo de diseñar soluciones para adaptarse al entorno. Las soluciones surgen de la dinámica de la familia-organización por interactuación con el consejero. Esta persona puede centrarse en procesos concretos o mucho mejor en el funcionamiento interactual de los subsistemas familia-propiedad-empresa de modo integrado, micro y macroanalíticamente, con conocimiento de las dinámicas familiares, desafíos emocionales y laborales, (Bork, et al., 1997).

El consejero debe definir previamente la familia empresaria como un sistema, conformado por al menos tres subsistemas (familia-empresa-propiedad) dentro de los cuales operan los familiares constituyentes ejerciendo diferentes roles. Para el Consejero la familia empresaria es una organización compleja, en la que una actuación de un constituyente reverbera en los tres subsistemas. La familia empresaria, como organización, debe ser concebida como un sistema que busca satisfacer varios objetivos, siendo los más importantes: conservar y acrecentar el patrimonio familiar y hacer competitiva las empresas de la familia. El Consejero debe buscar el equilibrio teleológico entre familia y empresa, sabiendo que los subsistemas están en un equilibrio dinámico y evolutivo a lo largo del tiempo, (Carlok y Ward, 2010)
Para sobrevivir a corto plazo la empresa familiar debe explotar de modo eficiente los recursos de que dispone, entre ellos, el conocimiento. Esta explotación eficiente demanda claridad en los objetivos y cierta estabilidad en su definición; normas, significados, roles y habilidades. Sin embargo, la familia empresaria, como tal organización, debe sobrevivir a largo plazo y para ello debe explorar el entorno, identificar oportunidades, pero esa exploración es un proceso que conlleva ambigüedad y supondrá requerir nuevos roles y habilidades. Esta explotación y exploración es una combinación dialéctica dentro de la familia empresaria. El consejero debe atender al diseño de un mecanismo cognitivo que conecte ambas funciones que consumen recursos y que tienen enfoques diferentes de corto plazo (explotación) y de largo plazo (exploración). La distancia cognitiva que exista entre familiares va a determinar la combinación de ambas funciones. Esa distancia se materializa en el distanciamiento que los familiares tienen a la hora de inferir racionalmente, percibir, interpretar, emitir juicios de valor $y$ experimentar emociones y sentimientos, pues cada uno construye su estructura cognitiva desde sus propias vivencias y experiencias. Es lo que se denomina dispersión del conocimiento dentro de la familia empresaria. El aprendizaje cognitivo es diferente y cuanto más distanciamiento cognitivo haya entre personas más difícil será lograr la colaboración. Pero cuanto mayor sea ese distanciamiento entre estructuras cognitivas más oportunidades existirán de explorar nuevas oportunidades. El consejero en su tarea tendrá que encontrar la transacción conveniente entre explotación y exploración para lograr el objetivo de supervivir en el mercado y como familia empresariaorganización (Riding y Rayner, 2005).

La confianza y la comunicación es fuente para crear una estructura cognitiva colectiva en el seno de la familia empresaria a fin de lograr un consenso o un pacto para definir objetivos y asignar recursos para conseguir esos objetivos. A través de la confianza y la comunicación se produce una estructura cognitiva familiar dinámica, vista desde una perspectiva coevolutiva, (Kepner, 1983) y esta confianza y

Rodríguez Zapatero, M., Rodríguez Jiménez, M. y Rodríguez Alcaide, J.J. (2012). El consejero en la empresa familiar: teoría y praxis. Revista de Empresa Familiar, 2(1), 45-54. 
comunicación se origina e influye biyectivamente en una determinada escala de valores y de creencias colectivas en el seno de la familia empresaria que facilitan el consenso y el compromiso de acción, (Dyer y Handler, 1994; Morris et al., 1997). La confianza está asociada a los atributos individuales de competencia, consistencia, responsabilidad, juego limpio, generosidad, benevolencia. La conectividad y la comunicación nace del mutuo respeto entre familiares con minimización de rivalidad, hostilidad y hostigamiento $\mathrm{y}$ acercamiento $\mathrm{y}$ acortamiento de distancias cognitivas. El acuerdo está moderado por el grado de inteligencia relacional entre familiares (Rodríguez et al., 2010).

La cohesión familiar está moderada por la confianza mutua entre familiares y por la comunicación interactiva. Esa confianza se fundamenta a nivel individual en los procesos vividos de reciprocidad, compromiso y lealtad de los componentes familiares, muy relacionados con la consuetudinariedad, la hermandad y el altruismo. Pero también esa confianza a nivel de familia empresaria nace del cumplimiento de ciertas normas, fijación de estándares justos y de normas éticas. Por fin, la confianza nace de las fuentes generales como son la cultura y la religión. La naturaleza y límites de la confianza están nítidamente expuestos en Nooteboom y Six (2003) y la confianza como generador de cohesión está desarrollada en (Bachmann, 2003).

La cohesión no existirá si no hay previamente coherencia entre hechos $y$ significados y sobre todo porque la cognición es un proceso que se construye mutuamente en interacción con otros. La cohesión exige una cognición colectiva diferente a la de cada familiar.

La cohesión nace de una cognición colectiva en la familia empresaria, red de procesos conectados de modo evolucionista. La cohesión nace partiendo del análisis de variadas perspectivas y no de un enfoque monocular; es decir, la cohesión surge de que los familiares quepan bajo el amplio enfoque utilizado.

\section{Metodología}

Las siguientes páginas explican el proceso de asesoramiento realizado con las familias empresarias. El proceso empleado queda definido por cuatro etapas consecutivas en el tiempo que quedan recogidas en la figura 1 .

\subsection{Primera etapa: introducción conceptual reglada.}

Hemos actuado a la demanda de familias empresarias una vez que estas familias empresarias han tomado un Seminario de 48 horas, durante 16 semanas, desde Octubre a

\section{Figura 1}

Diagrama procesal de asesoramiento.

$\begin{array}{lll}\text { Primera fase } & \text { Segunda fase } & \text { Tercera fase }\end{array}$

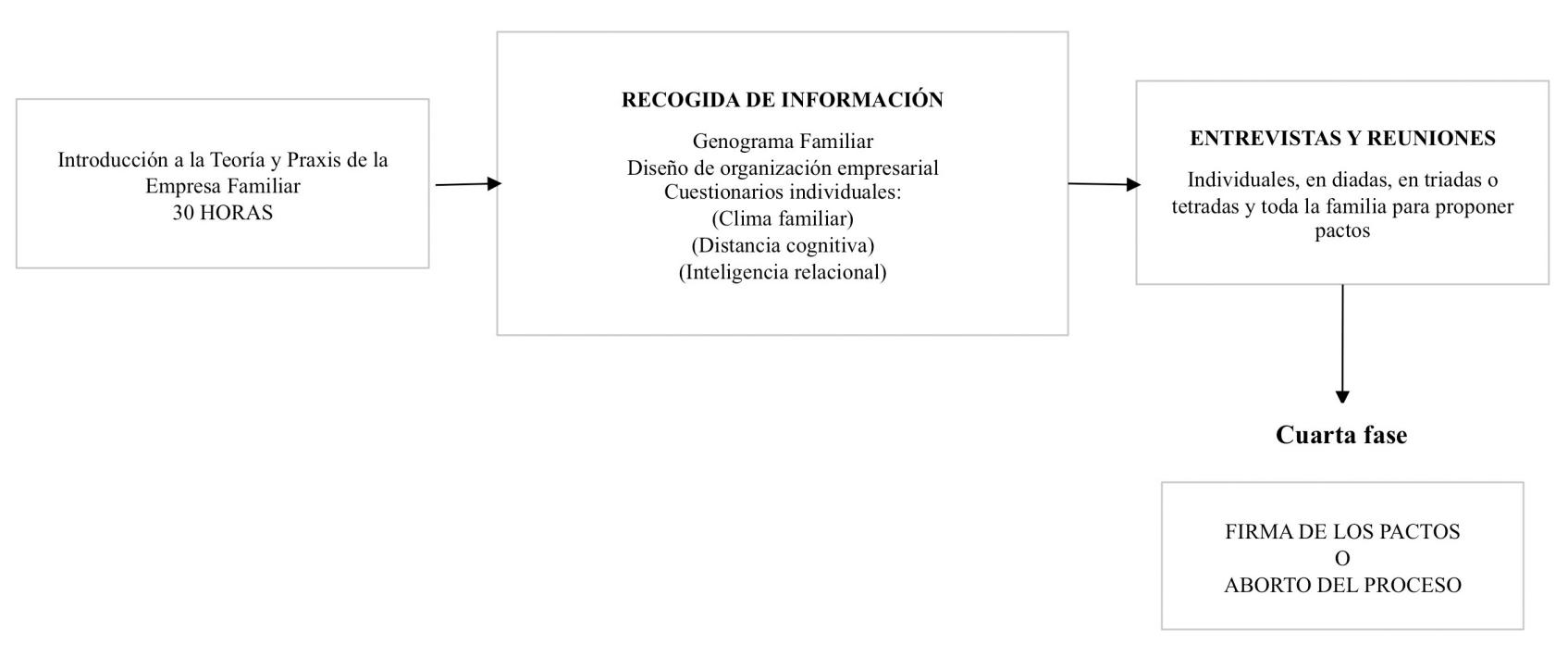

Rodríguez Zapatero, M., Rodríguez Jiménez, M. y Rodríguez Alcaide, J.J. (2012). El consejero en la empresa familiar: teoría y praxis. Revista de Empresa Familiar, 2(1), 45-54. 
Abril; es decir, desde el otoño hasta la primavera. El contenido del curso aborda diferentes temáticas, enfoques y cuestiones.

El Curso se estructura en 4 módulos. Cada módulo aborda los siguientes contenidos: Modelo de los 3 círculos desde el enfoque estático de Tagiuri (1982) y desde el enfoque dinámico de Gersick (1997). Sistema de valores y planificación paralela de la familia y la empresa de Carlock y Ward. El liderazgo familiar en empresas de primera y siguientes generaciones siguiendo a Solomon (2010). El patrimonio familiar y estrategias de conservación siguiendo a (Hughes, 2007). Al Seminario deben asistir los propietarios, los familiares de sangre, trabajen o no en la empresa familiar, y los familiares por afinidad que trabajen en la empresa y aquellos que, aún no trabajando, quieran voluntariamente atender las enseñanzas.

El fundamento de este enfoque es que la estructura cognitiva se irá descubriendo, revelando y reconfigurando a medida que se avanza en la información estructurada y en el curso de los períodos de reflexión a los que se obliga mediante la cumplimentación de cuestionarios. Esta estructura cognitiva se revela a nivel individual y no como colectivo (familia empresaria organización). Otro fundamento para esta pedagogía es la de conseguir que pausadamente se vaya creando un ambiente de confiabilidad de los familiares respecto del Consejero-profesor, en su caso, y de que este profesor vaya abriendo el enfoque cognitivo de todos y cada uno de los familiares respecto de la familia empresaria, organización holística, complejo de al menos tres subsistemas entrelazados: familia, empresa, propiedad.

A la hora de descubrir este nuevo enfoque, nuestra experiencia muestra el devenir de la misma desde enero del año 2000 hasta abril del año 2011; es decir, de prácticamente los últimos once años. Por esta experiencia han pasado 150 familias; de las cuales 53\% estaban en fase de fundador con hijos trabajando en la empresa, $45 \%$ en fase de sociedad fraternal con hijos trabajando en la empresa y $2 \%$ de sociedad de primos hermanos sin hijos trabajando en las empresas propias. De las 150 empresas solo 26 han solicitado ayuda para iniciar el camino hacia el pacto familiar en relación al futuro de la empresa familiar en sus diferentes apartados

Terminado este período de aprendizaje de la teoría o teorías sobre la familia empresaria y la empresa familiar, desde un enfoque académico y práctico o desde una perspectiva científica y el realismo, las familias pueden o no decidir que el profesor asuma el papel de consejero de la familia empresaria para abordar el camino a seguir en el futuro que permita trabajar juntos, queriéndose unos a otros, para que la empresa familiar crezca y superviva y genere beneficios, además de dar satisfacción a las necesidades básicas de los familiares, bajo el principio en la familia de "a cada cual según sus necesidades" y el principio de la empresa "a cada cual según contribuya".

\subsection{Segunda etapa:pasos previos antes de} decidir el acuerdo entre familia empresaria y consejero

La familia, que decide solicitar el apoyo del consejero, tras haber interiorizado todo el elenco de situaciones, conceptos, significados que para su familia ha supuesto la asistencia al seminario, y haber somatizado la dinámica evolutiva de familia, propiedad y empresa, debe cumplimentar, a nivel individual, unos cuestionarios específicos que permiten al consejero conocer las creencias, mitos, significados, enfoques, comprensiones, ambigüedades de la que extraer su estructura cognitiva y las distancias que, ante ciertas propuestas, cognitivamente las familias revelan. Desde un enfoque global el consejero obtiene el genograma de la familia extendida o de la familia múltiple y también una representación esquemática de la organización empresarial como empresa mononegocio, multinegocio o cartera de negocios, así como la estructura de la propiedad desde la empresa individual, pasando por el grupo de empresas, hasta alcanzar al holding si existiera.

Con los diferentes cuestionarios individuales de los componentes de la familia, desde el enfoque cognitivo, primariamente se analizan 
los datos para obtener una imagen de la familia en relación a los siguientes aspectos:

2.2.1. El triángulo del clima familiar, mediante el cual, siguiendo a Bjömberg y Nicholson, (2007), determinamos la posición de cada familiar en los espacios "emoción-cognición", "comunicación-adaptabilidad" y "atención personal y diálogo intergeneracional". Este instrumento tiene su fundamento en las siguientes proposiciones: (1) las interacciones entre familiares generan un determinado clima familiar, (2) que la familia empresaria es un sistema adaptativo y solucionador de problemas siguiendo los enfoques de Olson et al. (2003) y Beavers et al. (1983) y (3) que la familia es un sistema de regulación sicológica (Moos, 2002) $\mathrm{y}$, por último, que la familia empresaria para abordar problemas y resolverlos necesita cohesión; que a su vez depende de la capacidad cognitiva y emocional de los constituyentes (Rodríguez y Rodríguez, 2006).

Para cada familia ubicamos a sus componentes en el espacio "cohesión cognitivacohesión emocional", a fin de esclarecer la distancia cognitiva que existe entre los componentes de la familia que depende no solo de cada familia sino de la etapa generacional en que la familia empresaria se encuentra. Igualmente se localiza cada familiar en el espacio "comunicación mutua-adaptabilidad" que no tiene como moderador la etapa generacional de la empresa familiar. Este análisis ayuda al entendimiento del clima familiar.

2.2.2. La distancia cognitiva entre familiares respecto del sistema familia y el sistema empresa se pone al descubierto mediante un cuestionario bipolar que el familiar cumplimenta para así medir la distancia de cognición que entre ellos muestran. En relación a la empresa el consultor indaga la distancia que muestran los familiares respecto a las siguientes concepciones.

- La empresa familiar como un negocio que debe mantener alto grado de eficiencia financiera, buena imagen ante la sociedad, la búsqueda de valor como criterio de actuación.
- La empresa familiar como artefacto para promover el cambio, la asunción del correspondiente riesgo y deseo de profesionalidad.

- La empresa, como sistema que ambiguamente se orienta por el binomio (lealtad versus mérito" y el binomio "seguridad en el empleoretribución variable".

Así mismo en relación al sistema familia el Consejero indaga la distancia que los familiares muestran respecto de las siguientes concepciones:

- La familia empresaria se mueve hacia un estilo de dirección autoritario o hacia un estilo de dirección participativo en la empresa, con peso objetivo y de mayor ponderación o no de los mayores.

- La familia ve la empresa como una oportunidad de desarrollo personal, como un empleo fijo y seguro y como un sistema de elevada flexibilidad normativa.

El consejero mediante este cuestionario observa la distancia cognitiva entre familiares y si esa distancia permite observar un equilibrio entre los dos subsistemas familia y empresa. Las distancias cognitivas más importantes son las siguientes:

- Entre empleo seguro y retribución fija y empleo seguro y retribución variable.

- Entre entrada de familiares a trabajar en la empresa sin restricciones o entrada mediante un proceso selectivo.

- Entre promoción basada en lealtad versus promoción basada en logros alcanzados y méritos.

- Entre considerar la empresa como un sistema regulado por normas o un sistema muy flexible.

- Entre creación de valor empresarial, mantenimiento de excedentes y profesionalización frente a drenaje de recursos financieros y ausencia de transparencia.

- Entre estilo de dirección autoritario frente a estilo de dirección participativo de la empresa.

2.2.3. La inteligencia relacional como fundamento para lograr el consenso, a través de la confianza mutua y la comunicación, es otro elemento previo que el consejero utiliza para saber del pronóstico previo del posible éxito de 


\section{Figura 2}

Modelo básico para un acuerdo familiar.

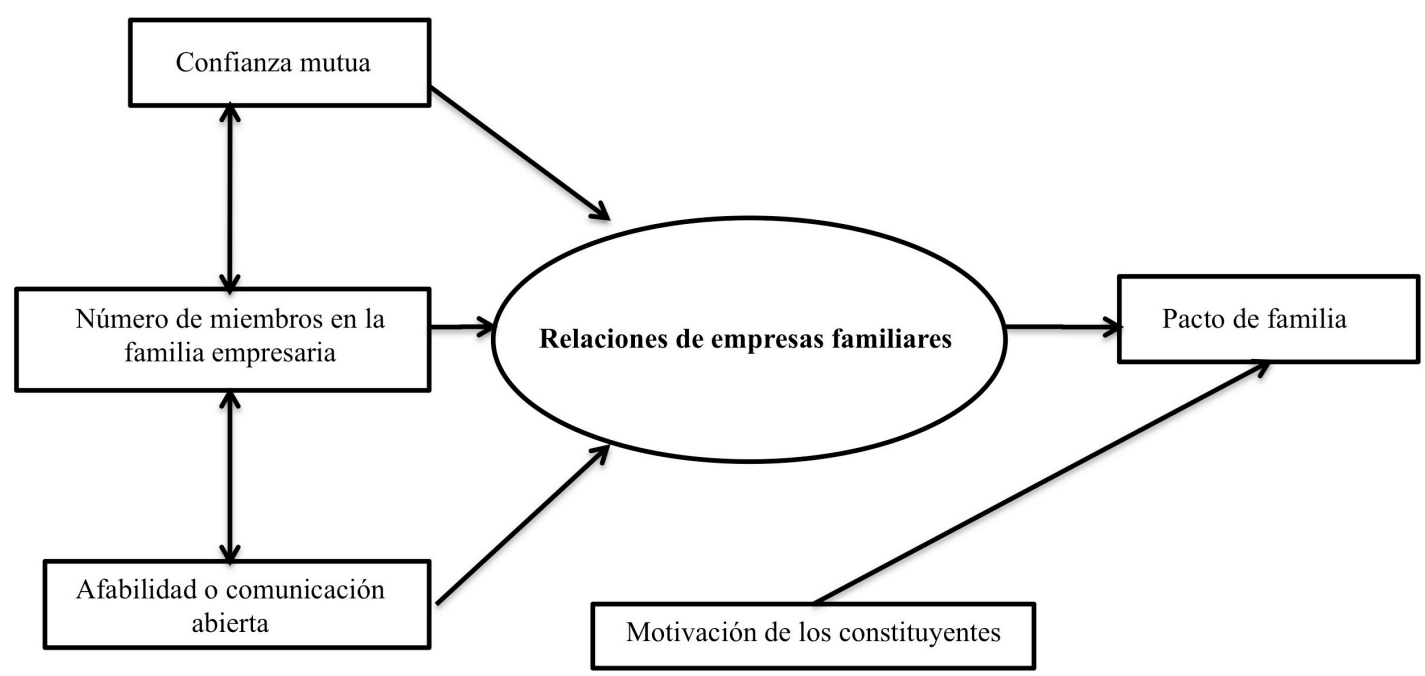

la tarea. El método utilizado se basa en el trabajo de Hoover y Hoover (1999), que nos ha dado un resultado excelente, nos permite posicionar a cada familiar en el espacio "confianza-habilidad de comunicación", de acuerdo con la capacidad intelectual y la inteligencia emocional de cada constituyente de la familia empresaria. La confianza es la transparencia, honestidad y ausencia de reservas mentales sobre la integridad de cada uno de ellos; esta confianza está asociada a atributos de competencia, consistencia, juego limpio, generosidad, benevolencia, (Morris et al., 1997). La comunicación y conectividad nace del mutuo respeto y de la minimización de la rivalidad, hostilidad y hostigamiento. El modelo utilizado ha sido extraido de (Rodríguez et al., 2010).

\section{La investigación y sus resultados}

Nuestro modelo de análisis aparece diagramado en la figura 2. Un pacto familiar tácito puede mantenerse en el tiempo o explicitarse con éxito para gerenciar la empresa familiar, si la motivación de los constituyentes es elevada y si las relaciones intercorpusculares del sistema gozan de unos atributos de elevada confianza mutua y elevada habilidad relacional, lo que a su vez depende del número de constituyentes en el juego y del estado de cada individuo y de los subsistemas.
Nuestra hipótesis es la siguiente:

H1: Si la confianza relacional es elevada así como la afabilidad de sus componentes la predictibilidad de alcanzar un pacto explícito es elevada.

H2: Si el número de constituyentes es elevado y la empresa está en fase de sociedad fraternal y/o de primos hermanos, el nivel de habilidad relacional disminuye y también el grado de confianza relacional por lo que la predictibilidad de alcanzar un pacto es baja.

En el presente estudio han participado 26 familias empresarias de 125 componentes en su totalidad, con un tamaño medio por familia de 5 miembros.

Esta experiencia nos indica que la variable que facilita el consenso es la comunicación, dado un nivel de confianza en la familia, verificado por un modelo logit (Rodríguez et al., 2010). La visión global de nuestro estudio se refleja en la figura 2.

El mapa de inteligencia relacional de estas 26 empresas aparece en la figura 3. Cada empresa está representada por una burbuja o círculo, cuyo área representa el nivel de motivación (Rodríguez et al., 2010) de la familia empresaria que evoluciona de 1 a 5 como intervalo de valoración y su posición en el espacio de coordenadas indica el cuadrante de inteligencia relacional (Rodríguez et al., 2010) en el que se 


\section{Figura 3}

Inteligencia relacional de las 26 empresas analizadas.

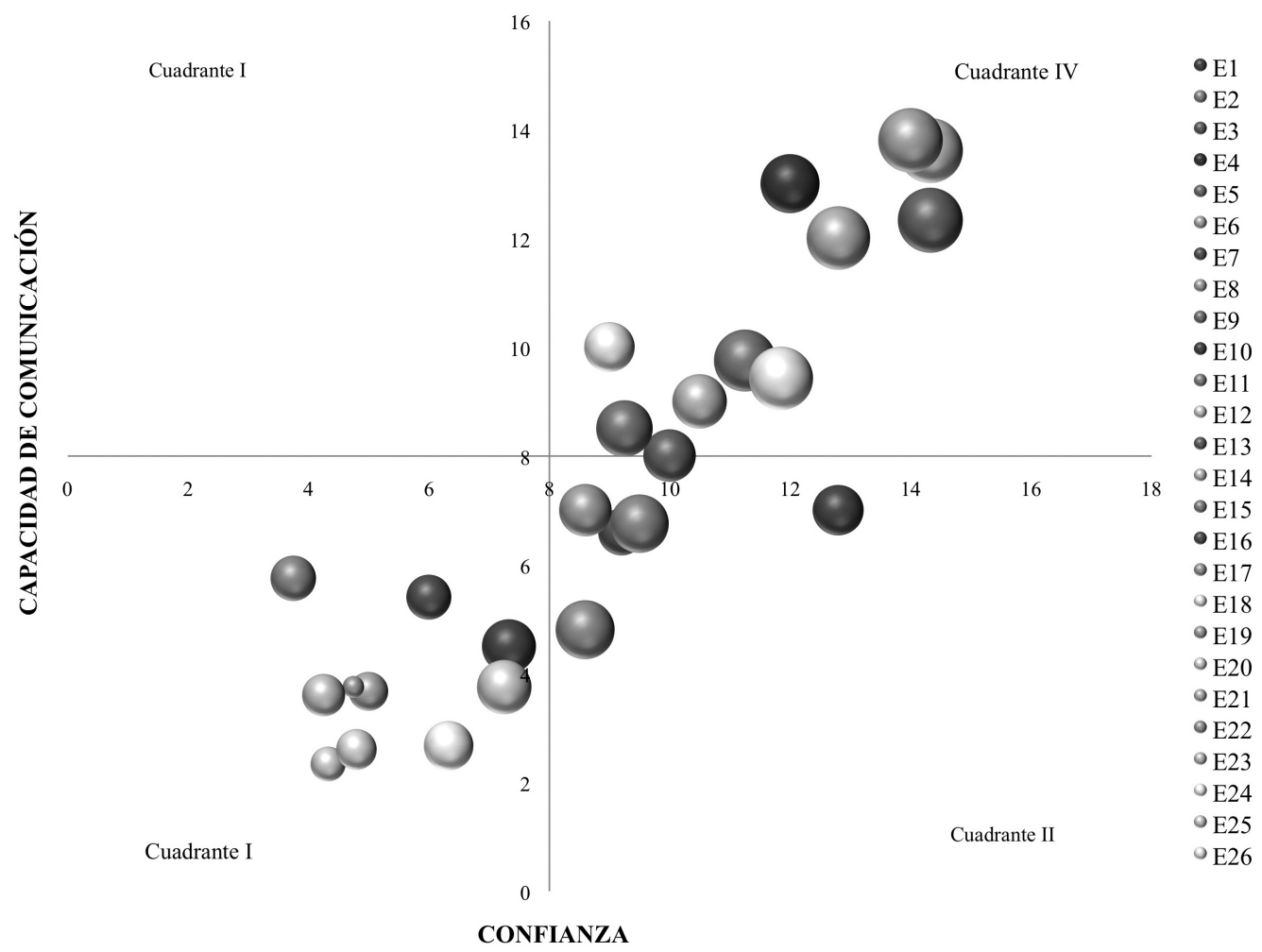

encuentra cada familia y que observamos en la figura 3 .

En la figura 3 se refleja que de las 26 empresas analizadas, 11 se encuentran en mejor cuadrante posible (IV), donde la confianza y la comunicación son altas y el valor de la motivación está por encima de la media, valor que se determina por el tamaño de la burbuja y que aparece reflejado en el interior de la misma junto al número de empresa. Diez empresas se encuentran en el cuadrante I; es decir, en el peor cuadrante posible, donde el paradigma y la capacidad son bajos y la motivación está por debajo de 3, excepto en dos de ellas en las que a pesar de exhibir una inteligencia relacional baja la motivación está por encima de 3.

Estas dos empresas con una motivación alta tendrán menos problemas que el resto para llegar al cuadrante deseado; es decir, a una inteligencia relacional alta, ya que tienen el motor necesario para ello.

Cinco empresas están en el cuadrante II lo que nos indica que tienen una confianza o paradigma relacional alto pero una baja habilidad relacional.

\section{Comprobación de hipótesis}

El objetivo del análisis de la información obtenida mediante esta encuesta es comprobar la hipótesis siguiente: la inteligencia relacional está correlacionada positivamente con la firma de un protocolo familiar discutido $y$ consensuado por todos los miembros de la familia empresaria.

En la tabla 1 se relaciona la posición media de la familia empresarial en los cuadrantes de inteligencia relacional con la situación respecto del protocolo.

Para cada familia el consejero analiza la inteligencia relacional del sistema familia empresaria. Las familias en primera generación, que están en el cuadrante de mayor confianza mutua y de mejor comunicabilidad y mayor motivación, llegan al pacto con más probabilidad que si, ubicados en ese cuadrante óptimo, se encuentran en segunda generación. 
Tabla 1

Relación entre Inteligencia relacional y situación del protocolo.

\begin{tabular}{lllll} 
& $\begin{array}{l}\text { Proceso } \\
\text { finalizado }\end{array}$ & En proceso & $\begin{array}{l}\text { Proceso } \\
\text { abortado }\end{array}$ & $\begin{array}{l}\text { Ném } \\
\text { empresas }\end{array}$ \\
\hline IV & 7 & 3 & 1 & 11 \\
III & - & - & - & 0 \\
II & 1 & & 4 & 9 \\
I & 3 & 1 & 6 & 10 \\
\hline Total & 11 & 4 & 11 & 26 \\
\hline$\%$ & $42,31 \%$ & $15,38 \%$ & $42,31 \%$ & $100 \%$ \\
\hline
\end{tabular}

\subsection{Tercera etapa: el proceso de apoyo y mediación}

Tras proporcionar las dos primeras etapas en una cartografía de la familia

y de sus constituyentes respecto de la distancia cognitiva, inteligencia relacional y clima cognitivo emocional, se procedió a entrevistar individualmente a cada familiar mediante un procedimiento semiestructurado, cuyo diseño depende de la generación del entrevistado y roles que juega en la familia y la empresa. Las entrevistas proporcionaron información para evaluar sus expectativas, miedos, deseos personales y clarificar algunas posiciones cognitivas así como rivalidades y conflictos de carácter cognitivo. Terminado el período de entrevistas individuales se inician entrevistas conjuntas por generación $\mathrm{y}$ entre generaciones a fin de acotar y reducir las distancias cognitivas y aclarar posicionamientos previamente identificados y evaluados.

Las cuestiones estructurales a analizar tanto a nivel individual como de subgrupos y de familia son:

- Mecanismos de gobierno de la familia extendida.

- Gobierno de las empresas.

- Acceso del familiar a la empresa y promoción.

- Retribuciones y compensaciones.

- Derechos y obligaciones de los propietarios.

- Resolución de conflictos.

4.2. Cuarta etapa: formalización del pacto familiar

La formalización del pacto, que se supone a partir de una visión integrada de las etapas de

\section{Figura 4}

Árbol de ramificación de la experiencia consultora.

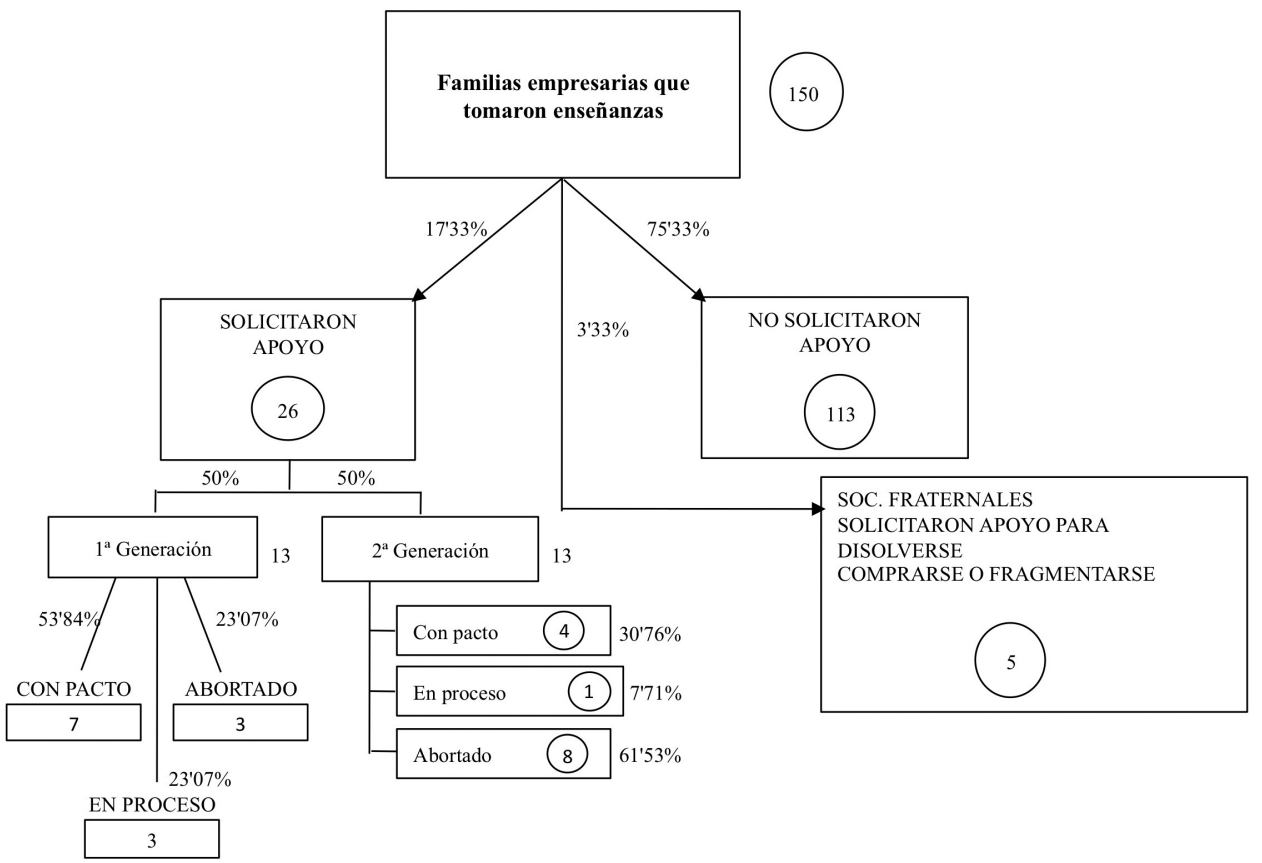


desarrollo concreto de las familias, su empresa o empresas y de cada individuo, lleva tiempo de reflexión, recogida de información y diálogo antes de llegar a la formalización del pacto. La experiencia avala cuanto aquí se expresa y queda reflejada en el árbol de ramificación de la figura 4.

De 150 familias empresarias del mismo entorno social, en que prácticamente casi la mitad de ellas estaba en fase de fundadordirector y algo más de la mitad en fase de sociedad de hermanos, los resultados muestran que tres cuartas partes no acudieron a nuestro asesoramiento para abordar el futuro del sistema de modo holístico. Lo hicieron el 3'33\% en fase de sociedad de hermanos para disolverse, vender la empresa o comprarse unos a otros o fragmentarse. Solo acudieron a nuestra ayuda el $17^{\prime} 33 \%$ de la población de familias empresarias para solicitar nuestra ayuda a fin de alcanzar un pacto sobre el futuro. Es destacable que el $53{ }^{\prime} 84 \%$ de las que estaban en fase de primera generación con hijos trabajando en la empresa formalizaron el pacto y el $23 \%$ lo abortaron, estando en proceso el resto; justamente lo contrario sucedió en el caso de empresas en segunda generación de los que solo el $300^{\prime} 76 \%$ firmaron el pacto y $61 ' 53 \%$ lo abortaron. El tiempo en alcanzar el pacto depende del nivel de inteligencia relacional, cohesión cognitiva $\mathrm{y}$ emocional, relación intergeneracional. Así pues ese tiempo de reflexión en nuestra experiencia fluctúa entre algo menos de de un año y tres años de conversaciones, períodos de reflexión, renovación de los ánimos, compromiso. (Rodríguez et al., 2010).

\section{Conclusiones}

La función del consejero familiar es ayudar a definir y consensuar el futuro de la familia empresaria, los caminos a tomar, los roles que cada familiar quiere tomar para alinearse con el proyecto y los recursos intelectuales y humanos que la familia ha de aportar compromiso (Rodríguez et al., 2010)

Para ayudar eficientemente a la familia empresaria en esta tarea el Consejero Familiar debe tomar en consideración lo siguiente:
- Que cada individuo de la familia está en disposición de no querer cambiar su opinión por el poder avasallador de sus propias convicciones frente a la realidad que perciben sus sentidos. No se debe olvidar que las convicciones heredadas configuran el modo de ver el futuro propio y el de la familia. Además, en gran parte el futuro se fabula recomponiendo el pasado; por ello, el Consejero Familiar no puede obviar el pasado de la familia empresaria y de cada familiar.

- El cerebro detesta cambiar de costumbres porque en ese cambio se juega la supervivencia del individuo, cuando paradójicamente en el cambio y adaptación al entorno hay un camino para la supervivencia. El Consejero Familiar no debe olvidar que podemos cambiar de opinión pero odiamos hacerlo y mostramos resistencia. Científicamente se ha demostrado que el cerebro cortical se bloquea cuando recibe informaciones disonantes, pues estas atentan contra la convicción. (Damasio, 1995)

- La disonancia cognitiva o el distanciamiento cognitivo es en realidad un conflicto entre dos ideas simultáneas y contradictorias que están sobre el tapete, lo que crea en cada individuo, en mayor o menor grado, desasosiego y estrés. Los circuitos cerebrales se inhiben para no evaluar ni ponderar esa disonancia. El Consejero Familiar puede ser el más disonante para algunos familiares y debe prestar atención a esta disonancia porque, tomada una decisión por alguien, se hace difícil cambiarla, lo que está comprobado neurológicamente.

- El Consejero Familiar debe saber, siguiendo a Damasio (1995) que "no hay proyecto que valga, sin emoción". Debe observar la emoción propia y de la familia a la hora de abordar el proyecto de continuidad de la familia empresaria. Al mismo tiempo, debe saber que la emoción no puede hacer perder el control del proyecto.

- La familia no podrá imaginar el futuro sin recordar su pasado, pues futuro y pasado están entramados. La memoria no solo sirve para recordar lo que aconteció sino también para anticipar el futuro, así que el consejero familiar tiene que apelar al pasado para proyectar la 
familia al futuro; según Damasio (1995) neurológicamente se conoce el camino del pasado (corteza sensorial-sistema límbicotálamo y corteza prefrontal) y el camino del futuro (sistema límbico-corteza sensorialtálamo-corteza prefrontal).

- El consejero familiar tiene que descubrir las intuiciones de los familiares y no solo las razones, en esta tarea de ayudar. Se enfrentará a familiares cautelosos, que intentarán no equivocarse antes de decidir concertar y familiares intuitivos que intentarán acertar y apostarán por ciertos pactos aún sin tener claros razonamientos: El cerebro de los cautelosos y de los intuitivos estará no solo reflexionando racionalmente sino haciendo el mayor tiempo conjeturas sobre el futuro. (Damasio, 1995)

- La tarea de un consejero familiar no es la de preveer, predecir, ni pronosticar el futuro sino la de ayudar a la familia empresaria a conformarlo, sabiendo que es la familia la que decide la ruptura o la continuidad y que ello depende de todas las consideraciones que aparecen en este trabajo, algunas, incluso, modelizadas.

- No es posible aconsejar sin conocer al individuo, a la familia y a la empresa.

\section{Referencias}

Bachmann, R. (2003). Trust and power as means of coordinating the internal relations of the organizations: a conceptual framework en B. Nooteboom y F. Six. The Trust Process in Organizations. Cheltennham: E.E.

Beavers, W.R. y Voeller, M. (1983). Family models: comparing and contrasting the Olson circumplex model with the Beavers system model. Family Process 22 (1), 85-89.

Bjornberg, A. y Nicholson, N. (2007). The Family Climate Scales-Development of a new mesure for use in family business research.Family Business Review 20 (3), 229-246.

Bork, D. y otros. (1997). Working with family business, San Francisco: Jossey Bass Publishers.

Carlock, R.S. y Ward, J.L. (2010). When Family Business are Best. Palgrave: MacMillan.

Damasio, A.R. (1995). Descartes error: Emotion, Reason and the Human Brain. London: Picard.
Dyer, W.G y Handler, W. (1994). Entrepreneurship and family business: exploring the connections. E.T.P. 19 (1), 71-84.

Gersick, K, Davis, J. , McCollom, M. y Lansberg, I. (1997) Empresas familiares generación a generación. Madrid: McGraw-Hill Interamericana Editores, S.A.

Hoover, E.A. y Hoover, C.L.(1999). Getting along in family business: The relationship intelligence handbook. N.Y.: Routledge.

Hughes J.E. (2007). Family. The compact among generations. N. York.: Bloomberg Press

Kepner, E. (1983).The family and the firm; a coevolutionary perspective. Organizational Dynamics, 12, (5770).

Morris, M.N., Williams, R.O., Ablen, J.A. y Avila, R.A. (1997). Correlates of success in family business transitions. Journal of Business Venturing $12,385-401$.

Moss, R.S. y Moss, B.S. (2002). Family environment scale manual.Palo Alto Ca. Mind Garden.

Nooteboom, B. (2003). The trust process. en The Trust process in organizations. Edward Elgar. Cheltenham: Editores B. Nooteboom y F. Six.

Olson, D.H. y Gorall, D.H. (2003).Circumplex model of marital and family business, en F. Walsh, Normal family process, N.Y.: Guildford.

Riding, R. y Rayner, S. (2005). Cognitive Styles and Learning Strategies. London: David Fulton Publishers.

Rodríguez Alcaide, J.J. y Rodríguez Zapatero, M. (2006). La empresa familiar: implicación y logro de sus contribuyentes. Cuaderno de investigación 4. Córdoba: Cátedra de Empresa Familiar. Universidad de Córdoba.

Rodríguez Zapatero, M., Rodríguez Jiménez, M. y Rodríguez Alcaide, J.J. (2010). Interpretación de la familia empresaria. Cátedra de Empresa Familiar. Córdoba: Universidad de Córdoba.

Solomon, S.D. (2010). Liderazgo en la empresa familiar. Bilbao: Ediciones Deusto.

Tagiuri, R, y Davis, J.A. (1982). Bivalent attributes of the family firm. Cambridge, Mass.: Working paper, Harvard Business School. 